\title{
Production d'électricité : évolutions techniques et économiques
}

\author{
Jean-Pierre HUTIN, EDF R\&D
}

\begin{abstract}
Résumé : toutes les technologies de productions d'électricité ne sont pas identiques et autant que les coûts annoncés, c'est leur structure, les modalités d'usages possibles et la capacité de répondre aux exigences sociétales qui détermineront l'avenir de leur développement. On passe en revue les différentes filières crédibles : les combustibles fossiles, le nucléaire, l'hydraulique et les productions d'appoint dont, en particulier les énergies dites renouvelables.
\end{abstract}

\section{I - CONTEXTE}

L'enjeu énergétique des précédentes décennies, dans la zone Europe, était d'accompagner la forte croissance des besoins en garantissant un approvisionnement énergétique au moindre coût, sous le contrôle des Etats.

L'enjeu actuel est plutôt de proposer un approvisionnement compétitif dans un contexte de fortes incertitudes (quelle organisation des marchés, quelles prises en charge des contraintes environnementales, quelles évolutions des prix des énergies fossiles, ... ), sous le contrôle hybride et encore très incertain des marchés, des Etats et d'organisations supra-nationales telles que la Commission Européenne.

L'enjeu essentiel de demain pour les européens sera très certainement, plus encore que de limiter les émissions de $\mathrm{CO} 2$, celui d'assurer la sécurité des approvisionnements énergétiques, dans un contexte de rareté des énergies fossiles, dont ni le nucléaire ni les ENR ne pourront assurer la relève au niveau mondial. Si la demande énergétique mondiale évolue conformément à la variante basse de l'AIE, et même si un effort significatif est fait pour développer les énergies alternatives non carbonées, nous serons confrontés à la situation décrite dans le schéma ci-dessous si les réserves fossiles sont conformes à l'estimation moyenne (dont l'écart type est important toutefois) des spécialistes :

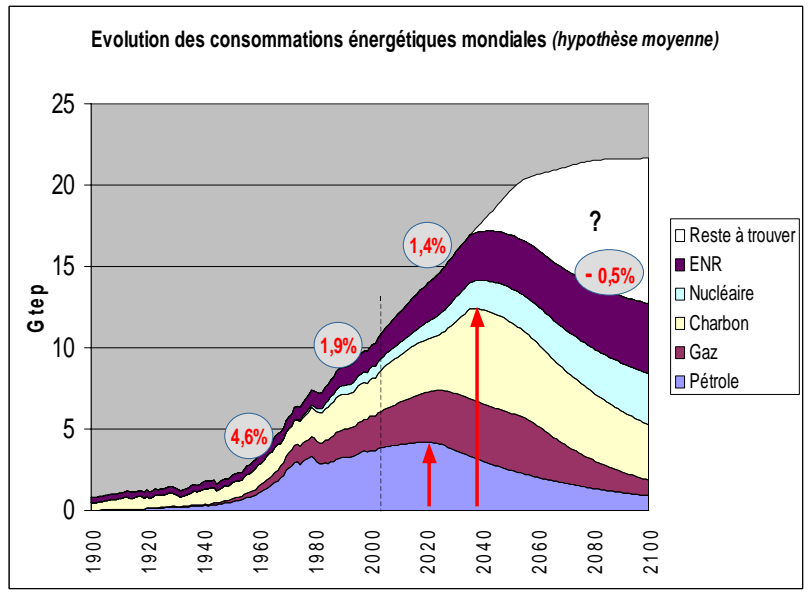

L'incertitude n'est pas dans la forme de la courbe, mais dans :

1. les dates des tensions pétrolières et fossiles. En fonction des réserves fossiles et de la demande, le peak oil pourrait intervenir entre 2010 et 2030 et le pic fossile entre 2020 et 2050

2. les solutions que la R\&D mondiale proposera pour "combler le blanc",

3. les bouleversements socio-économiques à venir...

\section{II - LES COMBUSTIBLES FOSSILES}

Toutes les études prospectives concluent que les combustibles fossiles resteront encore longtemps (au moins tant qu'il y en a !) une des sources d'énergie les plus utilisées, en particulier dans les pays en développement qui trouveront là une réponse évidente à des besoins grandissant très rapidement.

En ce qui concerne l'utilisation du charbon, les recherches en cours se concentrent sur la mise en oeuvre de nouvelles filières de production d'électricité à haut rendement capables de respecter des réglementations très strictes en matière d'environnement.

Les chaudières à charbon pulvérisé avec traitement des fumées

Les centrales à charbon pulvérisé modernes sont le produit de plus de 80 années de développements et $\mathrm{d}$ 'améliorations en matière de conception et, même si leur technique est ancienne, elles ont très bien su s'adapter à l'évolution de la réglementation sur les émissions polluantes et leur rendement n'a pas cessé de progresser.

Les nombreuses recherches actuellement en cours portent sur l'amélioration des brûleurs et sur une optimisation de la conception du foyer, pour minimiser la formation des oxydes d'azote, ainsi que sur le traitement aval des fumées qui concerne principalement la désulfuration, la dénitrification complémentaire et le dépoussiérage. Ces recherches, ainsi que l'emploi de cycles vapeur supercritiques à haut rendement, devraient permettre à la filière traditionnelle de combustion du charbon de rester en compétition avec les autres filières "charbon propre" pour la construction des futures centrales.

\section{$\underline{\text { Les Lits Fluidisés Circulant (LFC) }}$}

Dans cette technique, une masse importante de particules solides, généralement des cendres, est mise en suspension dans un foyer par un fort débit d'air. Cette masse chaude en mouvement, où sont injectées des particules de 
charbon et de calcaire, est continuellement entraînée vers l'extérieur du foyer et doit être séparée des fumées par un séparateur cyclone afin d'être recyclée. Cette recirculation dans le foyer permet une combustion satisfaisante du charbon, malgré la relativement basse température qui est maintenue à $850-900^{\circ} \mathrm{C}$ afin de favoriser la captation du dioxyde de soufre par la chaux et pour minimiser la formation des oxydes d'azote.

\section{La gazéification du charbon intégrée à un cycle combiné (IGCC)}

Dans cette filière, un gaz, produit par la gazéification sous pression du charbon (à l'air ou à l'oxygène), est refroidi puis épuré aussi soigneusement que possible afin de servir de combustible dans une turbine à combustion dont les fumées à haute température permettent de générer de la vapeur pour alimenter une turbine à condensation classique. Ce cycle combiné, alimenté par $\mathrm{du}$ gaz de synthèse, a la particularité d'utiliser au maximum la chaleur récupérée au cours du processus de gazéification et pendant le traitement du gaz. Tout ceci concourt au très bon rendement de l'ensemble, qui atteint environ $43-45 \%$ et qui pourrait encore évoluer en fonction des progrès attendus sur les turbines à combustion. Cette technique a aussi le grand avantage, malgré sa complexité, de réduire très fortement les émissions de polluants gazeux et de limiter la quantité de rejets solides, mais son coût reste encore très élevé et des incertitudes subsistent sur le montant des charges d'entretien, sur la disponibilité en exploitation et sur les possibilités de suivi de charge.

\section{Gaz ou charbon?}

Actuellement, les moyens de production d'électricité à base de gaz naturel semblent plus performants que les moyens de production à base de charbon principalement en terme de coût d'investissement, de rendement et d'émissions de polluants gazeux. Mais le charbon demeure indispensable dans le bilan énergétique de nombreux pays en particulier quand ils disposent de ressources locales bon marché et ce type de centrales devrait continuer à être installé de façon soutenue dans le monde. A long terme, le coût du gaz est incertain et les risques géopolitiques sont élevés. Le charbon, dans la mesure où son utilisation respecte l'environnement, aura donc encore un rôle important à jouer dans l'avenir grâce à ses réserves très abondantes.

Le LFC semble bien placé du point de vue du coût de construction, de la facilité d'exploitation et du passage en cycle supercritique. Il permet par ailleurs de brûler une gamme plus large de combustibles que les chaudières à charbon pulvérisé. Par contre, en cas de réglementation très stricte vis-à-vis des rejets gazeux, il restera moins performant qu'un traitement humide des fumées s'il veut garder sa simplicité d'exploitation et sa compétitivité.

Les tranches à charbon pulvérisé, brûlant un charbon international et disposant d'un traitement des fumées, peuvent s'adapter à une réglementation très stricte sur les rejets gazeux.
L'IGCC est la filière la plus performante en ce qui concerne le rendement et la protection de l'environnement. Elle a la particularité de fournir un produit intermédiaire, le gaz de synthèse, qui peut être utilisé dans l'industrie chimique ou dans les raffineries. Toutefois, son utilisation n'est envisageable que pour la production d'électricité en base. Pour la gazéification du charbon, son coût actuellement trop élevé devra être réduit par son industrialisation et par les progrès attendus sur les cycles combinés.

Par ailleurs, la nécessité de réduire fortement les émissions de $\mathrm{CO} 2$ à moyen/long terme pourrait conduire à capter et stocker le $\mathrm{CO} 2$ émis par les grandes installations de combustion dans des formations géologiques souterraines (principalement dans les anciens gisements d'hydrocarbures ou les aquifères profonds. Le coût du $\mathrm{kWh}$ serait évidemment très sensiblement augmenté (environ 8 centimes d'euros/kWh en base), en particulier à cause de la baisse globale du rendement et des investissements nécessaires.

La captation est un procédé bien maîtrisé techniquement (et industriel) dans le cas du gaz naturel, plus délicat dans le cas du charbon du fait des impuretés. Le transport ne devrait pas être un point de blocage, même s'il reste encore des incertitudes importantes à lever : corrosion interne des pipes, études de risques, transport par noria de bateaux...En revanche, la séquestration pose encore de nombreux problèmes : étanchéité des réservoirs, contrôle et monitoring pour évaluer les taux de fuites, limites acceptables, cadres juridiques (surtout pour les sites marins), impacts et risques pour l'environnement, responsabilité sur le long terme, etc...

Les caractéristiques des principales filières à combustibles fossiles sont résumées dans le tableau en annexe 1 (Ph. Jaud, EDF R\&D, 2005).

\section{III - LE NUCLEAIRE}

\section{$\underline{3.1 \text { - De la première à la quatrième génération }}$}

Le nucléaire constitue certainement l'une des réponses les plus crédibles au double défi énergétique et environnemental auquel nous serons confrontés dans les décennies à venir. Les orientations récentes prises par de nombreux pays sont le signe évident d'une relance qui confirme que le nucléaire est une solution d'avenir. Rappelons d'abord ce que sont les différentes générations de réacteurs nucléaires.

La génération I comprend les premiers réacteurs prototypes qui ont été mis en service avant 1970.

La génération II correspond aux premiers réacteurs commerciaux des années 1970 à 1995 dans les différentes filières REP, REB, VVER et Candu. Elle constitue 85\% $\mathrm{du}$ parc électronucléaire mondial en exploitation aujourd'hui (soit environ 450 réacteurs). Le retour d'expérience industriel de ces dernières décennies a permis de démontrer les performances tant économiques qu'environnementales de ces systèmes de production, 
avec un coût du kWh nucléaire très compétitif par rapport à celui des énergies fossiles et des progrès constants sur le niveau des rejets, déjà très inférieurs aux limites autorisées. Le fonctionnement cumulé de plus de 10.000 années-réacteurs au niveau mondial prouve la maturité industrielle de ces technologies.

La génération III correspond aux réacteurs avancés ABWR, AP600, EPR, AP1000 ou encore HTR modulaire. Les réacteurs de cette génération sont susceptibles d'être opérationnels avant 2010-2015.

Leur conception a intégré le retour d'expérience des générations précédentes : meilleure prise en compte du facteur humain, conception plus élaborée de la conduite, prise en compte des études probabilistes de sûreté, redondance accrue des dispositifs de sûreté, exécution passive des fonctions de sauvegarde, gestion en toute sécurité des conséquences d'un accident très sévère, etc. (en notant que nombre de ces améliorations ont été intégrées a posteriori dans les réacteurs de $2^{\text {ème }}$ génération existants).

La génération IV est celle des systèmes du futur : elle fait l'objet d'innovations et de développements importants, tant du point de vue du réacteur que du cycle du combustible, et devrait pouvoir être déployée au plus tard vers 2030. Les objectifs visés pour ces systèmes, de même que le choix des technologies clef pour les atteindre, font l'objet d'une coopération très active à l'international, notamment dans le cadre du Forum Generation IV.

Trois étapes ont déjà été franchies :

- l'évaluation, selon une méthodologie très codifiée, de concepts proposés par les pays participants,

- la sélection d'un petit nombre de concepts porteurs de technologies jugées particulièrement prometteuses,

- l'élaboration d'un plan de développement de ces technologies.

Quatre objectifs principaux ont été définis pour caractériser les systèmes du futur. Ils doivent être à la fois :

- durables : c'est à dire économes des ressources naturelles et respectueux de l'environnement en minimisant la production de déchets ;

- économiques : aux plans du coût d'investissement, du coût du combustible, du coût d'exploitation de l'installation et du coût de production du $\mathrm{kWh}$;

- sûrs et fiables : avec une recherche de progrès et en éliminant autant que possible les besoins d'évacuation de population à l'extérieur du site, quelles que soient la gravité d'un accident ;

- résistants vis-à-vis des risques de prolifération et aisément protégeables contre des agressions.

A l'issue d'un important travail d'analyse et d'évaluation mené par tous les pays membre du Forum, six systèmes nucléaires ont été sélectionnés, qui répondent de façon significative aux objectifs fixés.

SFR (Sodium-cooled fast reactor system) : réacteur rapide à caloporteur sodium
Ce concept de réacteur à spectre rapide est associé à un cycle fermé permettant le recyclage de l'ensemble des actinides et la régénération du Plutonium.

Deux options principales sont envisagées :

- la première, avec un retraitement de combustible métallique $(\mathrm{Zr})$ et une puissance unitaire intermédiaire de 150-500 MWe (concept de type PRISM ou IFR) ;

- la deuxième, avec un combustible MOX et une puissance unitaire élevée de 500 à 1200 MWe (réacteur de type EFR associé au retraitement PUREX).

Compte tenu de la maturité de la technologie sodium, les clés de la faisabilité du SFR sont les technologies du combustible porteur d'actinides et les procédés du cycle associés. Le concept à combustible oxyde pourrait être prêt pour un déploiement industriel dès 2035 .

\section{GFR (Gas-cooled fast reactor system) : réacteur rapide à caloporteur hélium}

Le GFR est un système à spectre rapide permettant le recyclage homogène des actinides avec un gain de régénération supérieur à 1 . Sa puissance est de 600 à $2400 \mathrm{MWth}$; il est refroidi à l'hélium en cycle direct ou indirect avec un rendement thermodynamique élevé $(48 \%)$. Les clés de la faisabilité du GFR sont la technologie du combustible et des procédés du cycle associés, ainsi que l'approche de sûreté. Le recyclage du combustible usé est envisagé avec un procédé pyrochimique ou hydro-métallurgique, potentiellement sur le site même du réacteur. Le GFR est un concept performant pour l'utilisation des ressources naturelles et la minimisation des déchets à vie longue.

\section{LFR (Lead-cooled fast reactor system) : réacteur rapide à caloporteur alliage de plomb}

Ce concept de réacteur à neutrons rapides fonctionne avec un cycle du combustible fermé. Plusieurs concepts sont envisagés: les concepts dits "battery" avec des puissances unitaires 50-100 MWe et une durée de cycle de 10-30 ans, les réacteurs de puissance de $1200 \mathrm{MWe}$, et les concepts modulaires de 300-400 MWe. Les combustibles peuvent être soit métalliques, soit des nitrures, et permettent le recyclage de l'ensemble des actinides. Les clés de la faisabilité du LFR sont les matériaux résistants à la corrosion par le plomb à 550$800^{\circ} \mathrm{C}$, les combustibles et les procédés du cycle associés, et des procédés de conversion innovants pour la production d'électricité ou d'hydrogène.

\section{MSR (Molten salt reactor system) : réacteur à sels fondus}

Le MSR est un concept à spectre épi-thermique qui utilise une solution de sels fondus pour combustible liquide et caloporteur. Le MSR intègre dans sa conception un traitement et un recyclage en ligne du combustible sur le même site que le réacteur. Le sel retenu pour le concept de référence $(1000 \mathrm{MWe})$ est un fluorure de sodium, zirconium et d'actinides. La modération des neutrons est obtenue par la présence de blocs de graphite traversés par le sel combustible. Les clés de la faisabilité du MSR sont la sélection du sel fondu, les matériaux résistants à la corrosion par ce sel à 
$800^{\circ} \mathrm{C}$, le traitement du sel usé, et un mode de couplage à un système de conversion pour la production d'électricité qui évite la perméation du tritium.

\section{SCWR (Supercritical water-cooled reactor system) : réacteur à eau supercritique}

Deux types de cycles de combustible sont envisagés pour le SCWR, ce qui correspond à deux versions différentes du concept : un réacteur à spectre neutronique thermique associé à un cycle du combustible ouvert et un concept à spectre rapide associé à un cycle fermé pour un recyclage de l'ensemble des actinides. Les deux options ont un point de fonctionnement en eau supercritique identique : pression de $25 \mathrm{MPa}$ et température de sortie du cœur de $550^{\circ} \mathrm{C}$ permettant un rendement thermodynamique de $44 \%$. La puissance unitaire du concept de référence est de 1700 MWe. Les clés de la faisabilité du SCWR sont les matériaux de structure et de gainage du combustible devant résister à la corrosion sous flux et eau supercritique, la stabilité en fonctionnement normal et la gestion d'accidents de dépressurisation. Le SCWR est crédité d'un bon potentiel de compétitivité économique.

\section{VHTR (Very high temperature reactor system) : réacteur à très haute température refroidi à l'hélium}

Le VHTR est un système à caloporteur gaz avec un cœur à spectre neutronique thermique et un cycle du combustible ouvert. Le VHTR fonctionne à très haute température (au-delà de $1000^{\circ} \mathrm{C}$ ) pour pouvoir produire de l'hydrogène par décomposition thermochimique de l'eau (cycle iode/soufre) ou décomposition électrolytique (électrolyse à haute température).

Le concept de référence a une puissance unitaire de 600 MWth et utilise l'hélium comme caloporteur. Le cœur est constitué de blocs prismatiques ou de boulets. Les clés de la faisabilité du VHTR sont les matériaux à très haute température, la réalisation de composants conçus pour ces très hautes températures, le développement de combustible à particules à enrobages de $\mathrm{ZrC}$ mieux adaptées que celles avec $\mathrm{SiC}$ aux très hautes températures. A ces enjeux s'ajoutent le développement des procédés de production d'hydrogène et du mode de couplage au réacteur

\section{2 - Le renouvellement du parc nucléaire d'EDF}

Une des caractéristiques essentielles du parc nucléaire d'EDF, constitué de 58 tranches nucléaires ( $63 \mathrm{GWe})$, est qu'il a, pour l'essentiel, été mis en service en dix ans : de 1980 à 1990. La perspective de son renouvellement se situe à partir de l'horizon 2020 - 2030 .

Le parc de production futur sera en service pratiquement jusqu'à la fin du $21^{\text {ème }}$ siècle. A cet horizon de temps, compte tenu des nombreuses et fortes incertitudes, la composition du portefeuille de production devra tenir compte de l'équilibre à rechercher entre les risques économiques et financiers attachés aux différents modes de production. Cependant, il apparaît que le nucléaire, si les coûts en sont maîtrisés, constitue une solution robuste.
Il est donc essentiel de garder ouverte la possibilité de remplacer le parc nucléaire actuel par du nucléaire.

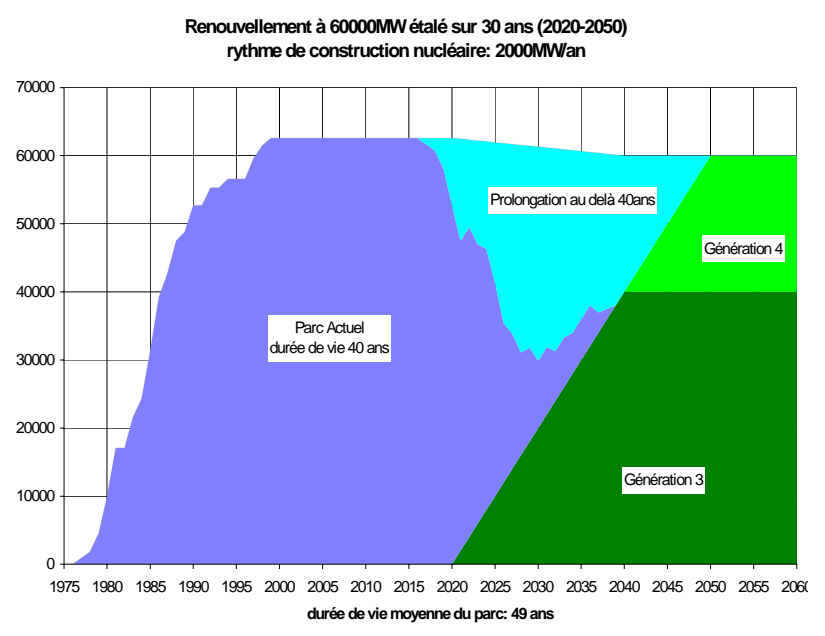

S'agissant des installations actuelles, EDF recherchera une durée de vie moyenne allant au-delà de 40 ans, modulée et différenciée selon les centrales. Ceci ne devrait pas poser de problèmes techniques rédhibitoires. Cependant, l'Autorité de Sûreté ne se prononcera pas sur ce point avant 2012/2015, la pratique française étant fondée sur un réexamen de sûreté décennal. Face à cette incertitude, il faut donc se mettre en situation de pouvoir engager dès 2015 (date de mise en chantier) le renouvellement par une filière nucléaire industriellement éprouvée.

S'agissant du rythme de renouvellement des centrales actuelles, les considérations industrielles et financières militent à l'évidence pour étaler dans le temps, le plus possible, la construction du parc futur. Il s'agit notamment de favoriser la flexibilité des choix successifs et la diversification du parc nucléaire futur, pour lui permettre d'accueillir, le moment venu, des modèles de nouvelle génération comme Generation IV. Un calendrier réaliste pourrait conduire à une maturité industrielle d'un système Generation IV à l'horizon 2040, la mise en service des centrales de génération III de type EPR se situant sur l'axe des temps entre 2020 et 2040.

La stratégie qui consisterait "à faire l'impasse" sur la génération III pour fonder le renouvellement du parc sur les systèmes Generation IV n'est guère envisageable en pratique. Elle supposerait de n'engager ce renouvellement qu'à partir de 2040 environ (année de mise en service). Il faudrait, pour cela, prolonger jusqu'à 60 ans, voire plus, la totalité des centrales actuelles et avoir la certitude de disposer en 2040 de technologies Generation IV totalement au point pour une introduction à un rythme très soutenu.

En résumé, l'enjeu de maintenir l'option nucléaire ouverte et de conserver une flexibilité maximale de ses choix, conduit EDF à prévoir le renouvellement de son parc de production en deux phases :

- une première phase à engager à partir de l'horizon 2020 sur la base de solutions de référence déjà éprouvées : réacteurs de génération III de type EPR, recyclage du 
combustible MOX, traitement du combustible usé à La Hague, conditionnement des déchets HAVL dans du verre et leur stockage après entreposage.

- une deuxième phase à l'horizon de 2040 sur la base de systèmes Generation IV, très probablement avec cycle fermé donc du type surgénérateur (réacteurs à neutrons rapides), en veillant à la cohérence des choix entre réacteurs et usines du cycle du combustible.

\section{IV - L'HYDRAULIQUE}

La production mondiale a été de 2645 TWh en 2000 , soit $18 \%$ de la production mondiale, pour une capacité installée de $700 \mathrm{GW}$. Le coût de production se situe entre 20 à $65 € / \mathrm{MWh}$ (coûts normatifs, très sensibles aux caractéristiques du site et aux taux d'actualisation).

C'est aujourd'hui la filière ENR la plus importante $(90 \%$ de l'électricité renouvelable), la seule pouvant répondre à de gros besoins et stockable de surcroît mais elle devrait, d'après l'AIE, diminuer car c'est une activité très capitalistique et de plus en plus critiquée sur le plan socio-environnemental. Les petits ouvrages ( $<25 \mathrm{MW}$ ?) sont peut-être les plus intéressants et accessibles. Il devrait quand même s'en construire 444 GW d'ici 2030 , les trois-quart dans les PED particulièrement en Chine.

Seuls 33\% du potentiel économique sont actuellement exploités. Le potentiel de développement serait donc de $5500 \mathrm{TWh}$. Mais l'énergie hydraulique n'est que l'un des aspects de la gestion globale de l'eau qui est peut-être le problème le plus important de la planète pour les 50 ans à venir. Un essor de cette filière ne pourra se faire sans intégrer tous les aspects de la gestion de cette ressource.

En France, la production hydroélectrique annuelle moyenne est de 69,8 TWh/an pour une puissance installée de $25200 \mathrm{MW}$ dont $4300 \mathrm{MW}$ pour les stations de pompage-turbinage. Le potentiel hydroélectrique théorique français est de $200 \mathrm{TWh} /$ an dont seulement 72 $\mathrm{TWh} / \mathrm{an}$ sont techniquement faisables. La production d'énergie hydroélectrique et le refroidissement des centrales thermiques à flamme et nucléaires conduisent déjà EDF à utiliser $75 \%$ des eaux de surface en France. Les possibilités de grands équipements nouveaux sont donc quasi-nulles.

La petite hydraulique est produite par 1700 usines totalisant $1972 \mathrm{MW}$ et produisant 4,6 TWh/an. EDF exploite environ 300 centrales, pour une production voisine de 3,6 TWh. Les possibilités du développement de nouvelles centrales sont limitées : d'une part, la plupart des sites intéressants sont déjà équipés et d'autre part, le classement d'un grand nombre de cours d'eau rend tout aménagement nouveau quasiment impossible pour l'instant.

Mais le véritable challenge de l'eau est de garder la possibilité de s'en servir ! L'eau représente une ressource primordiale pour EDF, que ce soit en terme de potentiel hydraulique ou comme fluide de refroidissement des centrales nucléaires ou thermique. Cette ressource est de plus en plus partagée avec des usages multiples (irrigation, tourisme, alimentation en eau potable). Les concessions de nombreux aménagements hydroélectriques et les arrêtés de rejet d'effluents de plusieurs centrales sont à renouveler dans un contexte de réglementations environnementales croissantes.

La canicule de l'été 2003, les tempêtes de fin 1999, les inondations récentes et multiples sont venues nous rappeler avec acuité le lien étroit de nos ouvrages de production avec cet élément naturel. La menace de changement climatique fait peser le risque d'une ressource en eau de plus en plus inégalement répartie dans le temps et l'espace : les évènements récents pourraient être les précurseurs du climat qui régnera vers 2050, des extrêmes en rareté ou en excès auxquels nous aurons à faire face.

Tout autant que la quantité, la qualité de l'eau fait l'objet de tensions et d'actions d'améliorations, à travers la Directive Cadre Européenne sur l'Eau, qui va durablement modifier les objectifs de gestion de l'eau des Etats Membres avec des résultats attendus en 2015.

\section{V - PRODUCTION D'APPOINT}

Sous l'appellation "production d'appoint", nous mettrons les technologies qui permettent une production locale de petite puissance, plus ou moins renouvelable et, surtout, plus ou moins aléatoire. Ces moyens de production ne pourront jamais assurer la production de base dont a besoin un pays développé. Par contre, ils doivent être pris en compte dans la vision que l'on peut avoir d'un système électrique du futur qui devrait intégrer un grand nombre de petites "centrales" produisant d'une façon peu prévisible.

\section{Microgénération}

De récents progrès technologiques sur les petits moteurs à gaz (à combustion interne ou externe comme le Stirling) laissent entrevoir l'apparition de moyens de cogénération (production combinée de chaleur et d'électricité à partir de gaz naturel ou d'autres combustibles liquides) à l'échelle de la maison individuelle. Cette cogénération à petite échelle est dite "micro-cogénération". Du fait de l'utilisation locale complète de l'électricité et de la chaleur produites, la micro-cogénération est un vecteur d'efficacité énergétique permettant de réaliser des économies d'énergie primaire.

Ces économies d'énergies primaires peuvent se traduire le cas échéant par une réduction des émissions de $\mathrm{CO} 2$ quand l'électricité cogénérée vient se substituer à un $\mathrm{kWh}$ " réseau » produit à partir d'énergie fossile. Les deux principaux moteurs de ce développement sont :

1. La réduction des impacts environnementaux,

2. La baisse de la facture énergétique globale (gaz + électricité) pour le client soit grâce à électricité autoconsommée ou soit grâce à la revente de la part non consommée sur place. 
Quatre technologies permettent de répondre au cahier des charges de la micro-cogénération :

- le moteur à combustion interne : rendement électrique de l'ordre de $20 \%$, technologie mature, des progrès récents, disponible aujourd'hui.

- le cycle de Rankine : rendement électrique de 10 à $15 \%$ ), des prototypes, concept simple, faible maintenance, disponible dans 3 ans.

- la pile à combustible : rendement électrique de 30 à $50 \%$, technologie pas encore mûre, faible maintenance attendue, pas de Nox \& CO, pas de bruit, disponible audelà de 5 ans.

- le moteur Stirling : rendement électrique de 10 à $15 \%$, technologie presque mûre (marine), faible maintenance, disponible dans 1 à 2 ans.

Pour la France, en considérant que ce type de technologie pourrait prendre à terme la moitié du marché de renouvellement des chaudières conventionnelles à horizon 2020, soit 3000000 machines (moteur à gaz puis moteur Stirling et Rankine), la production totale d'électricité serait de l'ordre de $8 \mathrm{TWh} / \mathrm{an}$. (à $75 \%$ de l'auto-consommation ).

\section{Le cas particulier des piles à combustible :}

Il existe différents types de piles à combustible, qui se distinguent principalement par leur température de fonctionnement et la nature de l'électrolyte (liquide ou solide) utilisé pour conduire l'électricité entre les électrodes (cf. tableau ci-dessous, où sont omis volontairement les PAFC (dépassées) et les DMFC, spécifiques aux applications portables). Dans le domaine de l'énergie, les piles à combustibles trouvent des applications dans le "stationnaire" (pour la cogénération ou la production d'électricité) et dans "l'embarqué", pour la propulsion des véhicules ou l'alimentation des périphériques.

\begin{tabular}{|c|c|c|c|}
\hline & $\begin{array}{c}\text { PetMic } \\
\text { (Proton Exchange Membrane Fuel } \\
\text { Cell) }\end{array}$ & $\begin{array}{c}\text { SOlC } \\
\text { (Solid Oxid Fuel Cell) }\end{array}$ & $\begin{array}{c}\text { MCIC } \\
\text { (Moliten Carbonate } \\
\text { Fuel Cell) }\end{array}$ \\
\hline $\begin{array}{l}\text { Température } \\
\text { de cellule }\end{array}$ & $20 \div 120^{\circ} \mathrm{C}$ & 200 a d $1000^{\circ} \mathrm{C}$ & $650^{\circ} \mathrm{C}$ \\
\hline $\begin{array}{l}\text { Rendement } \\
\text { Electrique }\end{array}$ & $35 \div 40 \%$ & $\begin{array}{l}45 \text { a } 50 \% \\
70 \% \text { se couplage Ple-turine (thytride) }\end{array}$ & 45 a $50 \%$ \\
\hline $\begin{array}{l}\text { Applications } \\
8 \\
\text { Combustibles }\end{array}$ & $\begin{array}{l}\text { siationaire électrogène } \\
\text { (H2, méthanol, GPL) } \\
\text { stationaire co-géneiration } \\
\text { gi membrane s } 120 \text { 'C (GN) } \\
\text { Trarsport: Traction } 8 \text { APU } \\
\text { (H2 ou diesel) }\end{array}$ & 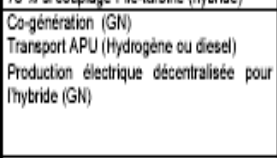 & $\begin{array}{l}\text { co-géééralion (GN } \\
\text { ou biogsz) }\end{array}$ \\
\hline Puistances & $\begin{array}{l}\text { de } 1 \text { a } 50 \text { WWe pour le siatornaire } \\
50 \text { a } 150 \mathrm{~kW} \text { pour le tranepot }\end{array}$ & 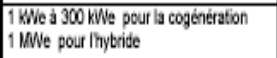 & $250 \mathrm{kWe}$ \\
\hline
\end{tabular}

Différents types de piles à combustible, source EDF R\&D

Malgré les annonces répétées d'avènement de cette technologie, les piles à combustibles tardent à arriver sur le marché. Pourtant, de réels progrès technologiques sont à l'œuvre comme en témoigne par exemple l'évolution de la densité de puissance des cœur de pile PEM, ou encore l'intensification des dépôts de brevet sur les piles à combustibles. Ces dernières années ont permis de passer de prototypes de laboratoire à de vrais démonstrateurs dont les performances sont bonnes, mais les coûts et durées de vie posent toujours problème.
En ce qui concerne l'usage microgénération stationnaire et partant des coûts actuels des systèmes $(20000 € / \mathrm{kW})$, 3 facteurs seront nécessaires pour atteindre le prix cible du marché :

- ruptures technologiques «produit » pour atteindre les performances adéquates : on pense que cette étape peut permettre aux piles d'atteindre $5000 € / \mathrm{kW}$ d'ici 3 à 4 ans. - rupture technologiques sur les procédés de fabrication : pour atteindre $3000 € / \mathrm{kW}$

- production de masse, qui seule pourra permettre d'atteindre un prix avoisinant $1000 € / \mathrm{kW}$

\section{$\underline{\text { Solaire photovoltaïque }}$}

Depuis près de 30 ans le domaine du photovoltaïque se focalise sur l'amélioration d'un même objet : les cellules au silicium cristallin à base de plaquettes. L'amélioration des procédés de fabrication de ces cellules et modules de première génération conjuguée à la croissance du marché du connecté réseau (croissance moyenne de 31\% par an sur les 10 dernières années) conduit à une décroissance régulière des coûts qui, si elle était maintenue, ne permettrait pas, malgré tout, au photovoltaïque de devenir compétitif (entre 350 à $1000 € / \mathrm{MWh}$ selon l'ensoleillement).

Cependant, deux ruptures technologiques sont aujourd'hui annoncées à moyen et long terme :

- les cellules en couches minces dites de deuxième génération : ces cellules sont fabriquées à partir de couches minces de semi-conducteurs, silicium, tellurure de cadmium (CdTe), ou diséléniure de cuivre et d'indium (CIS), déposés sur un substrat. Selon les experts, elle devrait supplanter la première génération à moyen terme c'est à dire d'ici 10 à 15 ans ;

- les concepts avancés à très haut rendement dits de troisième génération : ces cellules sont encore au stade de la recherche théorique. Elles offrent des perspectives à long terme, 30 à 50 ans, et concilient structures en couches minces et très hauts rendements de conversion pouvant atteindre $40 \%$ à $75 \%$.

Ces deux ruptures pourraient amener le coût de production de l'électricité photovoltaïque aux environs de 200 à $500 € / \mathrm{MWh}$ en 2010 voire $150 € / \mathrm{MWh}$ ou moins pour le PV couche mince après 2020.

\section{$\underline{\text { La biomasse }}$}

La production mondiale actuelle, pour l'essentiel non commerciale et utilisée pour les usages thermiques, est évaluée à 1,2 Gtep (dont 1 Gtep dans les PED) soit 14 $000 \mathrm{TWh}$. Les coûts de production varient entre 30 à 100 $€ / \mathrm{MWh}$ pour l'électrique, 10 à $40 € / \mathrm{MWh}$ pour la chaleur, 40 à $100 € /$ baril pour les carburants.

C'est une ressource qui a l'avantage d'être facilement substituable aux énergies fossiles (la seule qui puisse le faire pour les transports) ce qui en fait une option très intéressante, d'autant qu'elle a l'avantage d'être largement disponible dans les zones éloignées des centres de production. Elle est assimilable à une énergie de stock même si la ressource se constitue sous le flux des saisons. 
La biomasse représente un gros potentiel mondial mais pose de gros problèmes de concurrence d'usage des sols. Un autre point important est le fait que cette ressource n'est renouvelable qu'à condition d'être gérée correctement sur l'ensemble du cycle ce qui est rarement le cas aujourd'hui. La biomasse non commerciale qui représente aujourd'hui la plus grosse part de ressource prélevée est souvent un facteur de déboisement, voire de désertification.

En plus la production nécessitera de l'eau ce qui est à bien prendre en compte dans le bilan total dans un contexte où c'est aussi une ressource dont l'accès se réduit. Il en ressort qu'un développement important de cette filière ne peut pas s'envisager sans y intégrer des éléments de politiques agricole et d'aménagement du territoire.

La question de la neutralité de la filière en terme effet de serre est souvent discutée car il faut considérer tous les intrants (engrais) et l'énergie pour récolter et transporter puis transformer la biomasse, mais la substitution à des énergies fossiles qui est aisée reste bénéfique. Ce n'est donc pas la plus propre, mais elle se stocke.

Les évaluations de coûts (100 à $250 € /$ Tep) sont difficiles car selon qu'il s'agit de déchets à éliminer ou de cultures dédiées, de chaleur ou d'électricité, le coût complet change considérablement. Pour ce qui est de la production d'électricité, le coût de production dépend bien sûr beaucoup de la façon dont la biomasse est transformée (combustion directe et production d'électricité, méthanisation et production d'électricité, biocarburants).

\section{$\underline{\text { L'éolien }}$}

La production mondiale actuelle est de $606 \mathrm{TWh}$. Les coûts de production se situent entre 30 et $55 € / \mathrm{MWh}$ (coûts normatifs, très sensibles aux caractéristiques du site et aux taux d'actualisation ; progrès attendus : 20 $€ / M W h$ en 2020 ?). Pendant les années 90 le taux de croissance annuel a été de $15-20 \%$ : si cette tendance se poursuivait, on serait à $1200 \mathrm{GW}$ en 2020 pour couvrir jusqu'à $12 \% \quad(3000 \mathrm{TWh})$ des besoins en 2020 (25000TWh). Des scénarios plus raisonnables donnent une fourchette entre 300 à $1000 \mathrm{TWh}(100$ et $350 \mathrm{GW})$ en 2020 .

La filière a beaucoup progressé et est dès à présent compétitive dans certains cas, et les coûts devraient encore s'améliorer grâce à l'innovation, l'effet de série et l'industrialisation. Le potentiel mondial raisonnablement disponible serait supérieur à 50000 TWh annuels, si on inclut l'off-shore, soit plus de 4 fois la production mondiale actuelle d'électricité. Mais la croissance du marché sera en fait limitée par les possibilités d'adéquation entre le potentiel de ressource et la demande avec deux contraintes importantes :

- une insertion problématique dans les systèmes électriques et une acceptation incertaine,

- une production aléatoire.
Le caractère aléatoire, ou plus exactement non garanti, de l'éolien pose problème dans l'organisation actuelle des systèmes lorsque la part dans le "mix" électrique s'approche des $15 / 20 \%$. Il faut alors prévoir des moyens de stockage ou des moyens de pointe associés. L'intermittence des vents impose, si la part d'éolien devient importante, des capacités au moins triples par rapport à des équipements de base. Il est indispensable d'étudier ce que sont les potentiels de foisonnements de production éolienne sur de grands territoires. Ce qui est sûr c'est qu'utiliser ce potentiel suppose de faire évoluer les réseaux de transport et de distribution pour les adapter aux caractéristiques propre de l'éolien particulièrement en Europe où il se développe.

L'offshore, dont les coûts sont toutefois encore importants, soulage en partie ces contraintes : la production est plus stable et la gêne visuelle suscitée est moindre. Certains vont même jusqu'à envisager un couplage avec des "hydroliennes" (hélice placée sous l'eau pour récupérer l'énergie des courants de marée) pour mutualiser les coûts de génie civil et de raccordement aux réseaux terrestres.

\section{La géothermie}

On distingue trois sortes d'énergie géothermique fort différentes dans leurs manifestations et leurs utilisations : - Une géothermie moyenne et haute température, liée à la présence de roches chaudes peu profondes, destinée à produire directement de l'électricité à partir de gisements de vapeur ou d'eau chaude (centrale de Bouillante en Guadeloupe) ou après injection d'eau et récupération de chaleur (dispositif expérimental de Soultz sous forêt en Alsace).

- Une géothermie basse température alimentant un réseau de chaleur par l'intermédiaire d'échangeurs (exploitation de la couche du Dogger dans le bassin parisien).

- Une géothermie très basse température avec pompe à chaleur, utilisant la chaleur superficielle de la terre ou de nappes phréatiques, pour chauffer ou climatiser des locaux.

L'intérêt économique des centrales électriques géothermiques dépend beaucoup du gisement. Là où de la vapeur sèche ou de l'eau chaude à haute température est disponible en grande quantité et à faible profondeur, par exemple en Californie, au Mexique et aux Philippines, le système est rentable et des coûts de production sont de l'ordre de 0,03 euros/kWh. Pour les gisements de faible potentiel et de moyenne température, la petite taille des installations et les mauvais rendements rendent l'investissement cher, ce qui conduit à des coûts de production élevés $(0,08$ euros/kWh ?) qui nuisent beaucoup à son développement.

\section{Les énergies marines}

Que ce soit pour exploiter les courants (hélices placées sous l'eau et baptisées hydroliennes), les marées, les vagues ou les gradients de température entre la surface et les fonds, le potentiel maritime représente un potentiel énergétique renouvelable considérable surtout pour les régions côtières et les îles. Mais les coûts 
d'investissement et d'exploitation apparaissent durablement élevés.

\section{Le stockage de masse}

Demain, le métier des énergéticiens consistera à satisfaire des besoins diversifiés à partir d'énergies primaires devenues moins flexibles, plus dispersées et/ou plus intermittentes. Ceci supposera la capacité d'optimiser un système qui deviendra beaucoup plus complexe, où le stockage de l'énergie jouera un rôle clé.

Le seul procédé de stockage de masse couramment utilisé est le pompage de l'eau que l'on remonte dans les retenues en heure creuse pour qu'elle restitue son énergie par turbinage en heure pleine (STEP).

Il existe également des solutions de stockage électrochimiques qui possèdent des caractéristiques attractives et tendent à devenir économiquement intéressantes, mais les incertitudes technologiques et les coûts d'investissement élevés devraient limiter leur développement à des marchés de niches dans un premier temps.

En revanche, le stockage à air comprimé (CAES) est désormais capable de rivaliser avec le stockage hydraulique. Il s'agit d'une technologie mature, économiquement viable, dont l'adoption a certes été freinée par les forces du marché mais qui reste un bon candidat pour les applications du stockage de masse. Toutefois, à l'instar des STEP, il ne peut être implanté que sur des sites géologiques particuliers, ce qui peut représenter un frein à son développement.

Dans l'hypothèse d'un parc de production en développement sur les moyens de pointe avec une base surabondante, d'une prise en compte du coût des émissions de CO2 à hauteur de $20 € /$ tonne, les moyens de stockage type STEP (1000 MW) et CAES (490 MW) apparaissent compétitifs par rapport aux autres moyens de stockage et de pointe. Leurs faibles coûts de combustible et d'émissions de $\mathrm{CO} 2$ leur confèrent en effet un avantage significatif par rapport aux moyens de production de thermique classique (Turbine à combustion au gaz et fuel, unité de Charbon pulvérisé avec traitement de fumée et cycle combiné gaz).

Toutefois, il s'agit là de moyens fortement capitalistiques dont la durée d'amortissement est longue, ce qui peut gêner la décision d'investir dans un environnement incertain.

\section{Le stockage "local"}

Une batterie d'accumulateurs est un générateur électrique capable de convertir de l'énergie chimique en énergie électrique de manière réversible. L'énergie accessible d'une batterie dépend de la puissance appelée. Une batterie est aussi caractérisée par le nombre de charges et de décharges qui peuvent lui être appliquées.

Il existe 4 grands segments de marché pour ce produit - les batteries de démarrage des véhicules thermiques,
- les batteries industrielles pour le secours électrique, les chariots automoteurs, les outils portatifs et les applications ENR,

- les batteries d'équipements portables,

- les piles primaires pour les jouets, lampe de poche, radio etc...

En volume, les batteries représentent 30 Milliards de \$ chaque année pour $0,7 \mathrm{TWh}$. Les prix de vente des batteries s'échelonnent de $32000 € / M W h$ pour les batteries de démarrage à $320000 € / \mathrm{MWh}$ pour une batterie lithium-ion de téléphone portable!

Trois grandes technologies de batteries sont utilisées : les batteries à base de Plomb, les batteries à base de Nickel $(\mathrm{NiCd}, \mathrm{NiMH})$, les batteries à base de Lithium. Les marchés en croissance sont ceux du portable (téléphones, PC, PDA, ...) avec les batteries lithium-ion cobalt et dans un volume plus faible celui des véhicules hybrides équipés de batteries NiMH. L'accroissement des volumes de vente s'accompagne d'une baisse des prix mais ceuxci sont proches de leur asymptote.

Les autres marchés sont très matures avec des prix de batteries stables. La différence de prix entre les batteries au Plomb de démarrage et industrielle vient de la plus faible durée de vie des premières pour un volume d'énergie stocké puis déstocké donné.

\section{VI - ELEMENTS DE DISCUSSION EN GUISE DE CONCLUSION}

Toutes les productions d'électricité ne sont pas identiques et autant que les coûts annoncés, c'est leur structure ainsi que les modalités d'usages possibles qui comptent. En effet, leur valeur dépend de l'adéquation avec les besoins de la société et du respect de certaines caractéristiques de densité ou continuité de fourniture : les prix de fournitures fatales, de base ou de pointes sont différents. Par exemple, comparer directement le coût de production d'un $\mathrm{kWh}$ éolien imprévisible à celui d'un cycle combiné gaz n'a pas de sens si on n'y ajoute pas les coûts "systèmes" (transport, distribution, équilibre, garantie, etc.).

Les capacités de suivi de charge ou de stockage seront de plus en plus importantes pour un opérateur dont l'enjeu sera d'optimiser au mieux la valeur commerciale des kWh qu'il produira quel qu'en soit le coût de production initial.

Ne regarder que l'électricité ne suffit pas, il convient de considérer des co-productions électricité / chaleur. Enfin certaines productions peuvent avoir lieu chez le client final même, sans pour autant lui garantir l'autonomie et permettre de s'affranchir du réseau, ce qui complique les comparaisons.

Considérons les trois grandes familles de filières : fossiles, nucléaires et renouvelables.

Les filières fossiles : ce sont les spécificités du combustible qui importent. Même si des progrès incrémentaux restent encore possibles sur les centrales, 
les coûts de production seront fatalement tirés à la hausse par ceux des combustibles (surtout pour le gaz et le pétrole) et des nuisances environnementales induites (surtout pour le charbon et le pétrole).

Les filières nucléaires : les charges fixes constituent l'essentiel des coûts ce qui alourdit les coûts initiaux mais est un facteur de stabilité dans le temps. Les conditions d'exploitation (durée d'utilisation, variations de charge fréquentes) et le coût du capital (taux d'actualisation, temps de retour sur investissement) pèsent aussi beaucoup.

Les filières renouvelables : une diversité riche de promesses difficiles à tenir. Les ressources sont quasi inépuisables, les nuisances environnementales sont faibles ou réversibles (sauf pour l'hydraulique), et les potentiels de production sont considérables mais très localisés. Les coûts sont très variables et, pour les plus chères, des baisses importantes semblent possibles. Mais l'adéquation avec la demande finale restera toujours à faire : même si certaines productions peuvent se faire chez le client final, ces filières ne s'imposeront massivement qu'à condition de savoir gérer l'équilibre du système, donc fatalement avec un surcoût, sauf révolution dans le domaine du stockage, car par rapport à la plupart des usages :

- les flux de production sont déconnectés temporellement et géographiquement : il faudra pouvoir les transporter et/ou les stocker pour gérer leur intermittence.

- les densités de production sont très faibles : il faudra pouvoir les concentrer avant utilisation pour qu'elles puissent satisfaire les besoins finaux classiques. De ce fait, l'amélioration de l'efficacité énergétique finale favorisera leur développement.

Enfin, deux énergies "naturellement". stockables sont disponibles aujourd'hui où il y a des besoins (Pays en Développement) :

- l'hydraulique dont le potentiel économique est grand mais qui est de plus en plus difficilement acceptée.

- la biomasse qui a, surtout dans les zones rurales, un potentiel intéressant mais qui se heurtera vite à une forte concurrence d'usage des sols à cause de rendements très faibles.

\section{REMERCIEMENTS}

L'auteur remercie Bernard Rogeaux, Philippe Jaud et les chercheurs d'EDF R\&D pour leurs contributions.

\section{BIOGRAPHIE}

Après avoir étudié la mécanique en France et aux USA, Jean-Pierre HUTIN est entré à EDF en 1978 pour travailler sur le comportement des composants de centrales nucléaires. En 1991, il est devenu responsable du Département Maintenance Nucléaire et en 1995, il a été nommé directeur technique de la Division Production Nucléaire. En 2000, il a rejoint EDF R\&D comme Directeur de Programmes. 
Annexe 1 : caractéristiques des principales filières à combustibles fossiles

(Ph. Jaud, EDF R\&D, 2005)

\begin{tabular}{|c|c|c|c|c|c|}
\hline FILIERES & $\begin{array}{l}\text { CP + TF } \\
\text { (charbon) }\end{array}$ & $\begin{array}{l}\text { LFC } \\
\text { (charbon) }\end{array}$ & $\begin{array}{l}\text { PFBC } \\
\text { (charbon) }\end{array}$ & $\begin{array}{l}\text { IGCC } \\
\text { (charbon/rés. } \\
\text { pétroliers) }\end{array}$ & $\begin{array}{l}\text { Cycles } \\
\text { Combinés (gaz } \\
\text { nat.) }\end{array}$ \\
\hline $\begin{array}{l}\text { Etat de } \\
\text { développement }\end{array}$ & $\begin{array}{l}\text { Commercial } \\
\text { jusqu'à 1000-1300 } \\
\text { MWe }\end{array}$ & $\begin{array}{l}\text { Commercial } \\
\text { jusqu'à } 300 \\
\text { MWe } \\
\text { (600 MWe à } \\
\text { l'étude) }\end{array}$ & $\begin{array}{l}\text { Commercial } \\
\text { jusqu'à } 350 \\
\text { MWe pour les } \\
\text { lits fluidisés } \\
\text { denses sous } \\
\text { pression } \\
\end{array}$ & $\begin{array}{l}\text { Commercial } \\
\text { jusqu'à } 300 \\
\text { MWe pour le } \\
\text { charbon et } 500 \\
\text { pour les résidus } \\
\text { pétroliers }\end{array}$ & $\begin{array}{l}\text { Commercial } \\
1000 \text { MWe } \\
\text { et plus } \\
\text { (modularité) }\end{array}$ \\
\hline Point durs & $\begin{array}{l}\text { Broyage et séchage } \\
\text { du charbon, } \\
\text { encombrement des } \\
\text { dispositifs de } \\
\text { traitement des } \\
\text { fumées, rejets } \\
\text { liquides }\end{array}$ & $\begin{array}{l}\text { Grande quantité } \\
\text { de réfractaire, } \\
\text { risques } \\
\text { d'érosion, } \\
\text { utilisation des } \\
\text { cendres à } \\
\text { développer } \\
\end{array}$ & $\begin{array}{l}\text { Filtration des } \\
\text { gaz à chaud, } \\
\text { accessibilité } \\
\text { pour la } \\
\text { maintenance }\end{array}$ & $\begin{array}{l}\text { Nettoyage du } \\
\text { gaz complexe, } \\
\text { unité } \\
\text { cryogénique } \\
\text { pour la } \\
\text { préparation de } \\
\text { l'oxygène }\end{array}$ & $\begin{array}{l}\text { Durée de vie des } \\
\text { pièces chaudes, } \\
\text { nécessité d'un } \\
\text { combustible très } \\
\text { propre (donc } \\
\text { cher) }\end{array}$ \\
\hline Points forts & $\begin{array}{l}\text { Technique } \\
\text { éprouvée }\end{array}$ & $\begin{array}{l}\text { Simplicité en } \\
\text { exploitation, } \\
\text { combustibles } \\
\text { variés, pas de } \\
\text { rejets liquides }\end{array}$ & $\begin{array}{l}\text { Compacité, } \\
\text { augmentation } \\
\text { du rendement et } \\
\text { de la puissance } \\
\text { des tranches } \\
\text { existantes }\end{array}$ & $\begin{array}{l}\text { Rendement } \\
\text { élevé, bonne } \\
\text { protection de } \\
\text { l'environnement } \\
\text {, gaz de } \\
\text { synthèse } \\
\text { utilisable dans } \\
\text { la chimie } \\
\end{array}$ & $\begin{array}{l}\text { Rendement } \\
\text { élevé, faible } \\
\text { pollution, pas de } \\
\text { rejets solides, } \\
\text { construction } \\
\text { rapide, invest. } \\
\text { modéré }\end{array}$ \\
\hline $\begin{array}{l}\text { Rendement PCI } \\
\text { actuel à } 100 \% \text { de } \\
\text { charge }\end{array}$ & $45 \%$ & $39-40 \%$ & $42 \%$ & $45 \%$ & $58 \%$ \\
\hline $\begin{array}{l}\text { Rendement PCI à } \\
\text { l'horizon } 2015\end{array}$ & $(45$ à $50 \%)$ & $(45$ à $50 \%)$ & (46 à $51 \%)$ & (plus de $50 \%$ ) & (plus de $60 \%$ ) \\
\hline $\begin{array}{l}\text { Souplesse } \\
\text { d'exploitation }\end{array}$ & Moyenne & Bonne & Moyenne & Faible & Très bonne \\
\hline $\begin{array}{l}\text { Performances } \\
\text { environnementales }\end{array}$ & $\begin{array}{l}\text { Peuvent s'adapter } \\
\text { en fonction de la } \\
\text { réglementation }\end{array}$ & $\begin{array}{l}\text { Bonnes pour le } \\
\mathrm{SO}_{2}, \text { moyennes } \\
\text { pour les NOx }\end{array}$ & $\begin{array}{l}\text { Un peu } \\
\text { meilleures que } \\
\text { pour les lits } \\
\text { fluidisés } \\
\text { atmosphériques }\end{array}$ & $\begin{array}{l}\text { Très bonnes et } \\
\text { bien en dessous } \\
\text { de la } \\
\text { réglementation } \\
\text { actuelle }\end{array}$ & Excellentes \\
\hline $\begin{array}{l}\text { Coût } \\
\text { d'investissement } \\
\text { pour une unité de } \\
600 \mathrm{MWe} \text { avec } \\
\text { traitement des } \\
\text { fumées }\end{array}$ & $\begin{array}{l}1260 \text { Euro/kW } \\
(*)\end{array}$ & $\begin{array}{l}\text { Environ }-10 \% \\
\text { par rapport au } \\
\mathrm{CP}+\mathrm{TF} \\
(* *)\end{array}$ & $\begin{array}{l}\text { Environ }-10 \% \\
\text { par rapport au } \\
\mathrm{CP}+\mathrm{TF} \\
(* *)\end{array}$ & $\begin{array}{l}\text { Environ }+25 \% \\
\text { par rapport au } \\
\mathrm{CP}+\mathrm{TF} \\
(* *)\end{array}$ & $\begin{array}{l}550-650 \\
\text { Euro/kW } \\
(*)\end{array}$ \\
\hline $\begin{array}{l}\text { Coût de production } \\
\text { en base pour } \\
\text { une unité de } \\
600 \mathrm{MWe}\end{array}$ & $\begin{array}{l}\text { 3,35-3,95 cE/kWh } \\
\text { (suivant le prix du } \\
\text { charbon et le taux } \\
\text { de change du } \$ \text { ) }\end{array}$ & $\begin{array}{l}\text { Environ }-7,5 \% \\
\text { par rapport au } \\
\text { CP+TF pour du } \\
\text { charbon } \\
(* *)\end{array}$ & $\begin{array}{l}\text { Environ - } 5 \% \\
\text { par rapport au } \\
\text { CP+TF } \\
\text { pour du } \\
\text { charbon } \\
(* *) \\
\end{array}$ & $\begin{array}{l}\text { Environ }+25 \% \\
\text { par rapport au } \\
\text { CP+TF pour du } \\
\text { charbon } \\
(* *)\end{array}$ & $\begin{array}{l}2,75-4,25 \\
\text { cE/kWh } \\
\text { (suivant le prix } \\
\text { du gaz et le taux } \\
\text { de change du } \$ \text { ) }\end{array}$ \\
\hline
\end{tabular}

(*) Source : "les coûts de référence de la production électrique" rapport DIGEC de mai 1997. Avec un taux d'actualisation à $8 \%$ et pour une mise en service en l'an 2000.

(**) Il ne s'agit que d'estimations car aucune unité de 600 MWe n'a encore été construite. 